\title{
Statins attenuate but do not eliminate the reverse epidemiology of total serum cholesterol in patients with non-ischemic chronic heart failure
}

Authors: Hanna Fröhlich $a$, MD; Nandita Raman ${ }_{a}$; Tobias Täger ${ }_{a}$, MD; Dieter Schellberg ${ }_{a}$, MS; Kevin

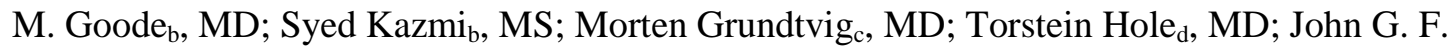

Cleland $_{\mathrm{e}}$, Prof.; Hugo A. Katus , Prof.; Stefan Agewall , Prof.; Andrew L. Clark , Prof.; Dan Atar

Prof.; Lutz Frankenstein ${ }_{\mathrm{a}}$, Prof.

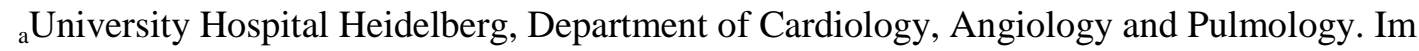

Neuenheimer Feld 410, 69221 Heidelberg, Germany. This author takes responsibility for all aspects of the reliability and freedom from bias of the data presented and their discussed interpretation.

${ }_{\mathrm{b}}$ Castle Hill Hospital of the University of Hull, Cottingham, HU165JQ, United Kingdom. This author takes responsibility for all aspects of the reliability and freedom from bias of the data presented and their discussed interpretation.

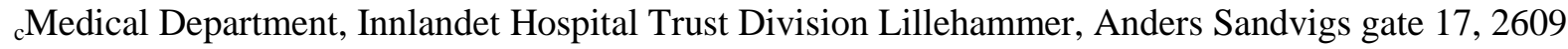

Lillehammer, Norway. This author takes responsibility for all aspects of the reliability and freedom from bias of the data presented and their discussed interpretation.

${ }_{\mathrm{d}}$ Medical Faculty, Norwegian University of Science and Technology (NTNU), Trondheim, Norway \& Medical Clinic, Helse Møre and Romsdal CHF, Åsehaugen 1, 6017 Ålesund, Norway. This author takes responsibility for all aspects of the reliability and freedom from bias of the data presented and their discussed interpretation.

eNational Heart \& Lung Institute, Royal Brompton \& Harefield Hospitals, Imperial College, SW72AZ London, United Kingdom. This author takes responsibility for all aspects of the reliability and freedom from bias of the data presented and their discussed interpretation.

${ }_{\mathrm{f}}$ Department of Cardiology, Oslo University Hospital, Ulleval and Institute of Clinical Sciences, University of Oslo, Kirkeveien 166, 0450 Oslo, Norway. This author takes responsibility for all aspects of the reliability and freedom from bias of the data presented and their discussed interpretation.

(C) 2018. This manuscript version is made available under the CC-BY-NC-ND 4.0 license http://creativecommons.org/ licenses/by-nc-nd/4.0/ 


\section{Corresponding author:}

Prof. Dr. med. Lutz Frankenstein

Im Neuenheimer Feld 410

69120 Heidelberg

Germany

Phone: 0049-6221-56-38895

Fax: 0049-6221-56-33575

Email: lutz.frankenstein@med.uni-heidelberg.de

Grant support/ Funding: This research did not receive any specific grant from funding agencies in the public, commercial, or not-for-profit sectors.

Conflicts of interest: Dr. Agewall reports personal fees from Advisory Board AstraZeneca, personal fees from Advisory Board Vifor, personal fees from Advisory Board Thermo Fischer Scientific, outside the submitted work.

Keywords: serum cholesterol, statins, reverse epidemiology, chronic heart failure, prognosis

Abbreviations: chronic heart failure (CHF), total serum cholesterol (TC), low-density lipoprotein cholesterol (LDL) 


\begin{abstract}
Background: In patients with chronic heart failure (CHF) increasing levels of total serum cholesterol are associated with improved survival - while statin usage is not. The impact of statin treatment on the "reverse epidemiology" of cholesterol is unclear.
\end{abstract}

Methods: 2,992 consecutive patients with non-ischemic CHF due to left ventricular systolic dysfunction from the Norwegian CHF Registry and the CHF Registries of the Universities of Hull, UK, and Heidelberg, Germany, were studied. 1,736 patients were individually double-matched on both cholesterol levels and the individual propensity scores for statin treatment. All-cause mortality was analyzed as a function of baseline cholesterol and statin use in both the general and the matched sample.

Results: 1,209 patients (40.4\%) received a statin. During a follow-up of 13,740 patient-years, 360 statin users $(29.8 \%)$ and $573(32.1 \%)$ statin non-users died. When grouped according to total cholesterol levels as low $(\leq 3.6 \mathrm{mmol} / \mathrm{L})$, moderate $(3.7-4.9 \mathrm{mmol} / \mathrm{L})$, high $(4.8-6.2 \mathrm{mmol} / \mathrm{L})$, and very high $(>6.2 \mathrm{mmol} / \mathrm{L})$, we found improved survival with very high as compared with low cholesterol levels. This association was present in statin users and non-users in both the general and matched sample ( $\mathrm{p}<0.05$ for each group comparison). The negative association of total cholesterol and mortality persisted when cholesterol was treated as a continuous variable (HR 0.83, 95\%CI 0.77-0.90, $\mathrm{p}<0.001$ for matched patients), but it was less pronounced in statin users than in non-users (F-test $\mathrm{p}<0.001)$.

Conclusions: Statins attenuate but do not eliminate the reverse epidemiological association between increasing total serum cholesterol and improved survival in patients with non-ischemic CHF.

Words: 245 


\section{Introduction}

Hypercholesterolemia is an established risk factor for the development of both cardiovascular disease and chronic heart failure (CHF) (1-3). In the general population, treatment of hypercholesterolemia with statins is effective in the primary and secondary prevention of major cardiovascular events, particularly when achieving very low levels of serum cholesterol $(1,2,4)$. In patients with CHF, however, the prognostic significance of hypercholesterolemia seems to change: numerous observational studies have found that low values of total serum cholesterol (TC) and low-density lipoprotein cholesterol (LDL) are associated with worse rather than better survival in patients with CHF (5-15). This counterintuitive risk inversion has been referred to as "reverse epidemiology" (13). It has been observed in both patients with acute and with chronic CHF of ischemic and non-ischemic origin (5-13). Conversely, lipid-lowering therapy with statins seems to be of no benefit in patients with $\operatorname{CHF}(1,2,16,17)$.

It is unclear to what extent treatment with statins contributes to the reverse epidemiology of serum lipids in $\operatorname{CHF}(8,10,12,18)$. On the one hand, statins lower TC levels which might be beneficial, yet in patients with $\mathrm{CHF}$, lower cholesterol levels may convey adverse prognosis. We therefore aimed to investigate the relative effects of statin treatment and TC levels on survival in a large international cohort of patients with non-ischemic CHF due to left ventricular systolic dysfunction.

\section{Material and Methods}

\subsection{Databases}

Patients' data were extracted from three different European CHF registries: the Norwegian Heart Failure Registry, the Heart Failure Registry of the Department of Academic Cardiology, University of Hull, United Kingdom, and the Heart Failure Registry of the University of Heidelberg, Germany. Recruitment was prospective and continuous for each database and center. All patients gave their written informed consent for data storage and evaluation. The study conformed to the principles outlined in the Declaration of Helsinki and was approved by the local ethics committees. 
The Norwegian Heart Failure Registry was initiated in October 2000 and patients were enrolled from outpatients' clinics of 27 recruiting hospitals well-distributed in all regions of Norway ranging in size and scope from small community to large university hospitals. The participating centers recorded their data using a web-based database.

Patients who attended the community heart failure clinics of the University of Hull, UK, and the University of Heidelberg, Germany, for evaluation of CHF were offered inclusion into the local CHF registries. The registries were initiated in 1999 and 1996, respectively. Since both university hospitals serve as primary health care centers as well as tertiary referral centers, the registries reflect a broad representation of patients of their respective regions.

\subsection{Patient selection and follow-up}

Patients were eligible for this study if they met all of the following criteria: a) attendance at the CHF outpatients' clinic of any of the participating hospitals, b) written informed consent for inclusion into the respective CHF registry, c) history of non-ischemic CHF due to left ventricular systolic dysfunction, and d) TC levels and medication recorded at the inclusion visit.

The diagnosis of CHF was established according to guidelines on the basis of typical symptoms and signs resulting from an objective abnormality of cardiac structure or function on echocardiography, cardiac magnetic resonance imaging, or left heart catheterization (19). Non-ischemic origin of CHF was verified by absence of a medical history of coronary heart disease or angina pectoris and absence of Q-waves from the electrocardiogram. When required, cardiac computer tomography, cardiac magnetic resonance imaging, or left heart catheterization was performed to exclude significant coronary heart disease. All included patients had a left ventricular ejection fraction $<45 \%$. Medication was at the discretion of the referring physician with respect to guideline recommended drugs.

Surviving patients were followed up for a minimum of six months. Determination of survival status and follow-up were performed by scheduled visits to the outpatients' clinic, by telephone calls either to the patients' homes or to their physicians, or by electronic hospital records. In addition, for the Norwegian Heart Failure Registry mortality data were obtained at regular intervals from the National 
Statistics Bureau, Statistics Norway. All-cause mortality was the pre-defined endpoint for the purpose of the analysis.

\subsection{Statistical analysis}

Calculations were performed using IBM SPSS statistics 20 (IBM, Ehningen, Germany) and MedCalc Statistical Software version 14.12.0 (MedCalc Software bvba, Ostend, Belgium). Graphs were displayed using GraphPad Prism version 6.00 for Windows (GraphPad Software, La Jolla California USA).

All tests are two-tailed and a P-value of less than 5\% was regarded as being statistically significant. Variables are presented as mean \pm standard deviation, median (interquartile range), or number (percentages; \%) as appropriate. Chi-squared test was used to compare frequencies. To test for significant differences between groups, the two-sample Wilcoxon test and Student's t test were used where appropriate. Differences in event-free survival were analyzed using Cox proportional hazard models and displayed using the Kaplan-Meier method for survival.

In order to analyze the relative effects of TC and statin treatment on survival, different strategies were applied:

First, survival was analyzed with respect to statin treatment in a univariable Cox regression analysis of the general sample. Then, patients were classified into 4 groups according to their $\mathrm{TC}$ at the inclusion visit defined as low $\left(\mathrm{TC}_{1} \leq 3.6 \mathrm{mmol} / \mathrm{L}\right)$, moderate $\left(\mathrm{TC}_{2} 3.7-4.9 \mathrm{mmol} / \mathrm{L}\right)$, high $\left(\mathrm{TC}_{3} 5.0-6.2 \mathrm{mmol} / \mathrm{L}\right)$, and very high $\left(\mathrm{TC}_{4}>6.2 \mathrm{mmol} / \mathrm{L}\right) \mathrm{TC}$. The graduation into 4 groups was chosen as a trade-off between sufficiently large a group size and enough groups to demonstrate the potential relationship between TC level and survival. Survival was compared between TC groups in the general sample, in patients treated with statins and in patients without statin therapy. In addition, survival within each TC group was analyzed with respect to statin treatment.

Second, TC was treated as a continuous variable, and the impact of TC on mortality was analyzed using Cox regressions. Again, analyses were performed in the general sample, in statin users and nonstatin users. 
Third, to account for possible confounders, a propensity score for the conditional probability of receiving a statin was derived for each patient. The propensity score was calculated as the single composite variable from a non-parsimonious multivariate logit-linked binary logistic regression of all baseline characteristics. The propensity score may be used to balance covariates, and numerous studies have reported that it is an adequate tool to reduce bias in observational studies (20-22). Statin treatment was the dependent variable (23). The logit of the probability of receiving a statin according to the score formed the basis of our matching procedure. Patients were individually matched on both the propensity of receiving a statin AND their TC levels. The matching procedure was performed in two steps: firstly, caliper matching of the propensity score was applied with caliper size predefined as 0.2 of the standard deviation of the total sample (24). In a one-pass procedure starting with a given patient receiving a statin, the closest match of a patient without statin therapy was identified. Secondly, TC levels were compared. If TC levels varied $\leq 10 \%$, the pair of patients was retained for analysis and removed from the total sample to allow for the next matching cycle to take place. If TC levels varied $>10 \%$, the pair was rejected. Then the first step of the matching process was repeated to identify the next closest match to the statin patients of the failed matched according to the propensity score. If a further patient without statin treatment was thus identified, the second step was repeated. In cases where we could find no match according to the propensity score AND TC level, the statin patient was removed from the total sample and the matching cycle started with the next statin patient.

All survival analyses were repeated in the matched sample. In addition, binary logistic regressions were performed to analyze the impact of TC on five-year mortality in matched statin users and nonusers. For graphic display, the logits of the regression models were used to calculate the relative probability of five-year death of each matched patient (statin users and non-users) with respect to TC levels following: probit $=1 /\left(1+e^{\wedge}\right.$-logit $)$. Curves were compared using the sum-of-squares F-test.

As a sensitivity analysis, the association of statin treatment, TC and mortality was analyzed in the general sample using stepwise multivariable Cox regression analyses. Variables that were significant in univariable analyses of the baseline characteristics were entered as covariates. Analyses were repeated in subgroups of statin users and non-users. In addition, as patients in the propensity score 
matched cohort differed with respect to age and country, survival analyses were repeated in the matched sample after adjusting for age and country using multivariable Cox regression analyses. Again, analyses were repeated in subgroups of statin users and non-users.

\section{Results}

\subsection{Patient characteristics and follow-up}

We identified a total of 2,992 patients who met the inclusion criteria outlined above. Of these, 1,616 (54.0\%), $893(29.8 \%)$, and $483(16.1 \%)$ patients were included from the Norwegian Heart Failure Registry, the Heart Failure Registry of the University of Heidelberg, and the Heart Failure Registry of the University of Hull, respectively. A total of 1,209 patients (40.4\%) was treated with a statin. Mean TC in patients without statin treatment was $5.2 \pm 1.3 \mathrm{mmol} / \mathrm{L}$, while it was $4.8 \pm 1.3 \mathrm{mmol} / \mathrm{L}$ in patients taking statins $(\mathrm{p}<0.001)$. Overall, patients on statins were older and more likely to suffer from comorbidities such as arterial hypertension and diabetes mellitus than patients without statin treatment. Baseline characteristics of the complete patient sample with respect to statin treatment are shown in table 1.

Total follow-up was 13,740 patient-years with a mean follow-up duration of 51.1 \pm 35.3 months (4.26 \pm 2.94 years). During that time, a total of 933 patients $(31.2 \%)$ died, $390(29.8 \%)$ in the statin group and $573(32.1 \%)$ in the non-statin group.

\subsection{Prognostic significance of statin treatment and $\mathrm{TC}$ in the general sample}

In univariable Cox regression analysis of the complete sample, treatment with statins was not associated with improved survival (HR 0.96, 95\% CI 0.84-1.10, $\mathrm{p}=0.59$ for use) (figure 1). When individual TC groups were analyzed separately, survival within each TC group was again not affected by statin treatment $(\mathrm{HR} 1.07,95 \%$ CI $0.77-1.48, \mathrm{p}=0.68$; HR $0.84,95 \%$ CI $0.67-1.04, \mathrm{p}=0.11$; HR 0.95, 95\% CI 0.75-1.21, $\mathrm{p}=0.70$; and HR 0.94, 95\% CI 0.65-1.37, $\mathrm{p}=0.76$ for use in $\mathrm{TC}_{1}, \mathrm{TC}_{2}, \mathrm{TC}_{3}$, and $\mathrm{TC}_{4}$, respectively). 
Mortality was significantly lower in patients with moderate, high or very high TC levels as compared to patients with low TC levels. This was true for patients on statins as well as for patients without statin therapy. In patients without statin treatment, however, there was no difference in survival between patients with low and patients with moderate TC levels (figures 2 and 3).

When treating TC as a continuous variable, Cox regression analyses found a significant negative association between TC and all-cause mortality in the general sample, in statin users and statin nonusers (HR 0.89, 95\% CI 0.85-0.94, p<0.001; HR 0.87, 95\% CI 0.80-0.94, $\mathrm{p}<0.001$; and HR 0.90, 95\% CI 0.84-0.96, $\mathrm{p}=0.001$, respectively).

\subsection{Prognostic significance of statin treatment and TC in the matched sample}

The matching procedure identified 868 pairs of statin/non-statin patients with equal TC levels and equal probability of receiving a statin. The matching significantly reduced standardized differences below $10 \%$ in the absolute values for the majority of observed covariates, demonstrating a substantial improvement in the covariate balance across the treatment groups. The baseline characteristics of the matched cohort are shown in table 2.

The analyses in the matched sample confirmed those from the general sample. Again, treatment with statins was not associated with improved survival in either the complete matched sample (HR 0.90, 95\% CI 0.75-1.07, $\mathrm{p}=0.24$ ), or in the respective matched TC groups (HR 0.97, 95\% CI 0.64-1.46, $\mathrm{p}=0.87$; HR $0.78,95 \%$ CI $0.59-1.03, \mathrm{p}=0.07$; HR $0.91,95 \%$ CI $0.66-1.25, \mathrm{p}=0.56$; and HR $1.23,95 \%$ CI $0.71-2.13, \mathrm{p}=0.46$ for use in $\mathrm{TC}_{1}, \mathrm{TC}_{2}, \mathrm{TC}_{3}$, and $\mathrm{TC}_{4}$, respectively).

As in the general sample, mortality was lower in matched patients with moderate, high, or very high TC levels as compared with matched patients with low TC levels (figure 4). Again, the association was seen in patients on statins as well as in patients without statin therapy, with the exception that in patients without statin therapy, survival did not differ between low and moderate TC levels (figure 5$8)$.

Treating TC as a continuous variable confirmed the negative relationship between TC and all-cause patients mortality in matched CHF patients (HR 0.83, 95\%CI $0.77-0.90, \mathrm{p}<0.001$ ). The association 
was less pronounced in matched patients with statin treatment as compared to non-users (HR 0.85, 95\% 0.77-0.95, $\mathrm{p}=0.004$; and HR 0.81, 95\% CI 0.73-0.90, $\mathrm{p}=0.0001$ for statin users and non-users, respectively).

Similarly, logistic regression analysis showed a significant inverse relationship between TC and fiveyear mortality in the common matched sample (OR $0.81,95 \%$ CI $0.74-0.90, p<0.001$ ), in matched statin users (OR 0.83, 95\% CI 0.72-0.96, p=0.01), and non-users (OR 0.79, 95\%CI 0.69-0.91, $\mathrm{p}=0.001$ ). The slope of the respective probability plots was less pronounced in statin users than in statin non-users $(\mathrm{p}<0.001)$. The probability of five-year death with respect to TC levels and statin treatment for matched CHF patients is displayed in figure 6.

\subsection{Sensitivity analyses}

Multivariable analyses of both the general and the matched sample confirmed the results from the main analyses: In stepwise multivariable Cox regression analyses of the general sample including significant variables from univariable analyses, statin treatment was not associated with survival $(\mathrm{p}=0.12$ ), while increasing levels of TC were associated with better survival (HR $0.89,95 \%$ CI 0.81 0.97, $\mathrm{p}=0.009$ ). This was true in statin users (HR 0.86, 95\%CI 0.74-0.99, $\mathrm{p}=0.049$ ) and non-users (HR 0.87, 95\%CI 0.77-0.98, $\mathrm{p}=0.02)$.

Similarly, stepwise multivariable Cox regression analyses of the matched sample including age and country as covariates did not find a relationship between statin treatment and survival (HR 0.88 , 95\%CI 0.74-1.05, $\mathrm{p}=0.14)$. Increasing levels of TC were associated with better survival in all matched patients (HR 0.83, 95\% CI 0.77-0.90, p<0.0001), matched statin users (HR 0.86, 95\% CI 0.77-0.96, $\mathrm{p}=0.006)$ and matched non-users (HR 0.80, 95\%CI 0.72-0.89, $\mathrm{p}<0.0001)$. Again, the relationship between TC and survival was less pronounced in statin users than in statin non-users. 


\section{Discussion}

We investigated the relative effects of statin treatment and TC levels on survival in an international sample of ambulatory patients with chronic CHF not due to ischemic heart disease. We observed a "reverse epidemiological" association between increasing TC and better survival irrespective of statin therapy where low TC levels were associated with worse survival as compared with moderate, high, or very high TC concentrations. Although statin therapy did not affect survival, statins attenuated the strength of the inverse association between TC and outcome.

Statins have become the most important lipid lowering medications with proven efficacy in the treatment of hyperlipidemia $(1,2)$. In addition to lowering cholesterol, statins appear to have pleiotropic effects which might influence pathophysiology and could potentially confer benefits on patients with CHF (25-27). The beneficial effects of statins have been attributed to their antiinflammatory properties, and their counter-regulatory actions on the renin-angiotensin-aldosterone and sympathetic systems as well as on the mechanisms of cardiac hypertrophy and fibrosis (26).

Treatment with statins in patients with CHF has been associated with reduced hospital admissions and mortality in several non-randomized studies $(25,28-31)$, post-hoc analyses $(32,33)$ and meta-analyses of randomized trials (34-36). The two largest randomized trials - the Controlled Rosuvastatin Multinational Study in Heart failure (CORONA) (16) and the Gruppo Italiano per lo Studio della Sopravvivenza Nell'Insufficienza Cardiaca Heart Failure study (GISSI-HF) (17) - however, failed to show a survival benefit of statins in patients with CHF. Current European guidelines therefore advise against statin therapy as a CHF treatment (1). The results of our study support this recommendation for patients with $\mathrm{CHF}$ due to non-ischemic etiologies, since survival was similar irrespective of statin treatment in both the general and the matched sample.

Our study also confirms the reverse epidemiological association between TC and survival in patients with chronic CHF. The phenomenon has been reported from numerous observational studies and posthoc analyses (5-15), and it has also been described for other cardiovascular risk factors such as obesity and hypertension $(11,13)$. The mechanisms underlying the risk inversion of classical risk factors in chronic CHF are presently unclear. In recent years, a number of plausible hypotheses has been 
proposed $(13,37-40)$ including the malnutrition-inflammation complex syndrome $(13,40)$ or theories focusing on endotoxin lipoproteins (37), ubiquinone (38), and selenoproteins (39).

Data regarding the influence of statin therapy on the reverse epidemiology are scarce. Silva et al. suggested that the low TC levels were not causally related to worse survival but simply represented an advanced stage of CHF. In a retrospective cohort study of 464 ambulatory CHF patients, they found that only patients with intrinsically low TC concentrations $(<3.9 \mathrm{mmol} / \mathrm{L})$ were at increased risk of death, while outcomes were best in CHF patients with pharmacologically induced low TC (12) - thus effectively arguing against the presence of reverse epidemiology in patients on statin therapy. Similarly, an Israeli study of 297 patients with advanced CHF reported that the negative association between LDL cholesterol level and mortality was only present in patients taking statins (18). In contrast, Kahn et al. reported that treatment with statins did not impact the reverse epidemiology of LDL in a study of 2,428 patients with acute CHF. (8).

In agreement with Kahn et al., we observed that low TC levels were associated with increased mortality as compared with high and very high TC levels irrespective of statin treatment. However, statins seem to attenuate the extent of risk inversion, since the negative relationship between TC and mortality was more pronounced in non-statin users. Whether this risk modulation is mediated by pleiotropic effects of statins remains speculative. Prospective trials are warranted to clarify the nature of risk inversion in CHF, the role of statins and possible treatment targets.

\section{Limitations}

A potential limitation to the present study is its observational design, implying the possibility of selection bias and unadjusted confounding. In addition, since matching was performed after treatment allocation, the relation between statin therapy, TC and all-cause mortality is associative, not causal. However, our data result from large comprehensive outpatient databases with continuous inclusion and close surveillance. Our study adds significant evidence to current knowledge by providing a large international patient cohort with a long follow-up and a robust statistical analysis. Notably, the 
presence of a reverse epidemiology of $\mathrm{TC}$ irrespective of statin treatment was established in univariable and multivariable regression analyses of both the general and the matched sample. Our data reflect the effects of TC levels and statin therapy in real-world patients in contrast to randomized trials. Our findings are clinically relevant to the population at interest given the known differences between study cohorts and "real world" patients.

We chose to restrict our analysis to patients with non-ischemic CHF since patients with ischemic CHF are usually treated with statins for coronary heart disease. Not only would this have hampered adequate matching to patients without statin therapy but the intrinsic prognostic benefit in coronary artery disease is unquestioned. A recently published observational study of 21,864 patients reported improved outcomes in statin users with CHF due to ischemic heart disease (31). Our results will therefore not be transferable to patients with CHF of ischemic origin. In addition, the classification as non-ischemic CHF may have been inexact in some patients since left heart catheterization was not performed in all patients. However, other studies observed a reverse epidemiology of TC in both ischemic and non-ischemic $\operatorname{CHF}(5,7,8,10-12)$.

The present study was limited by not having information on LDL levels. However, the "reverse epidemiology" has been reported for LDL as well as TC (5-15), and statins similarly affect both LDL and TC levels $(41,42)$. Therefore, it is likely that our results may be transferred to LDL measurements.

This study was further limited by not having information on the type of statin that was used in patients. As pharmacokinetics of lipophilic statins may differ from that of hydrophilic statins, a differential effect of certain statins cannot be ruled out $(43,44)$. Moreover, we cannot comment on medication adherence. Statin therapy may have been stopped during the course of follow-up. However, inclusion into the analyses of our study was performed after stabilization of both clinical status and medication in an ambulatory setting. This may reduce the necessity for further modulation of statin treatment. 


\section{References}

1. European Association for Cardiovascular P, Rehabilitation, Reiner Z, Catapano AL, De Backer G, Graham I, et al. ESC/EAS Guidelines for the management of dyslipidaemias: the Task Force for the management of dyslipidaemias of the European Society of Cardiology (ESC) and the European Atherosclerosis Society (EAS). European heart journal. 2011;32(14):1769-818.

2. Stone NJ, Robinson JG, Lichtenstein AH, Bairey Merz CN, Blum CB, Eckel RH, et al. 2013 ACC/AHA guideline on the treatment of blood cholesterol to reduce atherosclerotic cardiovascular risk in adults: a report of the American College of Cardiology/American Heart Association Task Force on Practice Guidelines. Journal of the American College of Cardiology. 2014;63(25 Pt B):2889-934.

3. Kenchaiah S, Evans JC, Levy D, Wilson PW, Benjamin EJ, Larson MG, et al. Obesity and the risk of heart failure. The New England journal of medicine. 2002;347(5):305-13.

4. Boekholdt SM, Hovingh GK, Mora S, Arsenault BJ, Amarenco P, Pedersen TR, et al. Very low levels of atherogenic lipoproteins and the risk for cardiovascular events: a meta-analysis of statin trials. Journal of the American College of Cardiology. 2014;64(5):485-94.

5. Greene SJ, Vaduganathan M, Lupi L, Ambrosy AP, Mentz RJ, Konstam MA, et al. Prognostic significance of serum total cholesterol and triglyceride levels in patients hospitalized for heart failure with reduced ejection fraction (from the EVEREST Trial). The American journal of cardiology. 2013;111(4):574-81.

6. Spinarova L, Spinar J, Vitovec J, Linhart A, Widimsky P, Fedorco M, et al. Gender differences in total cholesterol levels in patients with acute heart failure and its importance for short and long time prognosis. Biomedical papers of the Medical Faculty of the University Palacky, Olomouc, Czechoslovakia. 2012;156(1):21-8.

7. Horwich TB, Hernandez AF, Dai D, Yancy CW, Fonarow GC. Cholesterol levels and inhospital mortality in patients with acute decompensated heart failure. American heart journal. 2008;156(6):1170-6.

8. Kahn MR, Kosmas CE, Wagman G, Serrao GW, Fallahi A, Grady KM, et al. Low-density lipoprotein levels in patients with acute heart failure. Congestive heart failure. 2013;19(2):85-91. 
9. Afsarmanesh N, Horwich TB, Fonarow GC. Total cholesterol levels and mortality risk in nonischemic systolic heart failure. American heart journal. 2006;152(6):1077-83.

10. May HT, Muhlestein JB, Carlquist JF, Horne BD, Bair TL, Campbell BA, et al. Relation of serum total cholesterol, C-reactive protein levels, and statin therapy to survival in heart failure. The American journal of cardiology. 2006;98(5):653-8.

11. Guder G, Frantz S, Bauersachs J, Allolio B, Wanner C, Koller MT, et al. Reverse epidemiology in systolic and nonsystolic heart failure: cumulative prognostic benefit of classical cardiovascular risk factors. Circulation Heart failure. 2009;2(6):563-71.

12. Silva S, Lourenco P, Paulo C, Ferreira E, Lebreiro A, Sousa A, et al. Statin-induced low cholesterol is not associated with poor outcome in chronic heart failure. Journal of cardiovascular pharmacology and therapeutics. 2012;17(3):284-90.

13. Kalantar-Zadeh K, Block G, Horwich T, Fonarow GC. Reverse epidemiology of conventional cardiovascular risk factors in patients with chronic heart failure. Journal of the American College of Cardiology. 2004;43(8):1439-44.

14. Rauchhaus M, Clark AL, Doehner W, Davos C, Bolger A, Sharma R, et al. The relationship between cholesterol and survival in patients with chronic heart failure. Journal of the American College of Cardiology. 2003;42(11):1933-40.

15. Guder G, Gelbrich G, Edelmann F, Wachter R, Pieske B, Pankuweit S, et al. Reverse epidemiology in different stages of heart failure. International journal of cardiology. 2015;184:216-24. 16. Kjekshus J, Apetrei E, Barrios V, Bohm M, Cleland JG, Cornel JH, et al. Rosuvastatin in older patients with systolic heart failure. The New England journal of medicine. 2007;357(22):2248-61.

17. Gissi HFI, Tavazzi L, Maggioni AP, Marchioli R, Barlera S, Franzosi MG, et al. Effect of rosuvastatin in patients with chronic heart failure (the GISSI-HF trial): a randomised, double-blind, placebo-controlled trial. Lancet. 2008;372(9645):1231-9.

18. Charach G, George J, Roth A, Rogowski O, Wexler D, Sheps D, et al. Baseline low-density lipoprotein cholesterol levels and outcome in patients with heart failure. The American journal of cardiology. 2010;105(1):100-4. 
19. Ponikowski P, Voors AA, Anker SD, Bueno H, Cleland JG, Coats AJ, et al. 2016 ESC Guidelines for the diagnosis and treatment of acute and chronic heart failure: The Task Force for the diagnosis and treatment of acute and chronic heart failure of the European Society of Cardiology (ESC)Developed with the special contribution of the Heart Failure Association (HFA) of the ESC. European heart journal. 2016.

20. D'Agostino RB, Jr. Propensity score methods for bias reduction in the comparison of a treatment to a non-randomized control group. Stat Med. 1998;17(19):2265-81.

21. Rosenbaum PR, Rubin DB. The central role of the propensity score in observational studies for causal effects. Biometrika. 1983;70(1):41-55.

22. Rubin DB. Estimating causal effects from large data sets using propensity scores. Ann Intern Med. 1997;127(8 Pt 2):757-63.

23. Rubin DB. On principles for modeling propensity scores in medical research. Pharmacoepidemiology and drug safety. 2004;13(12):855-7.

24. Austin PC. Some Methods of Propensity-Score Matching had Superior Performance to Others: Results of an Empirical Investigation and Monte Carlo simulations. Biometrical J. 2009;51(1):171-84. 25. Bonsu KO, Kadirvelu A, Reidpath DD. Statins in heart failure: do we need another trial? Vascular health and risk management. 2013;9:303-19.

26. Ramasubbu K, Estep J, White DL, Deswal A, Mann DL. Experimental and clinical basis for the use of statins in patients with ischemic and nonischemic cardiomyopathy. Journal of the American College of Cardiology. 2008;51(4):415-26.

27. Correale M, Abruzzese S, Greco CA, Concilio M, Biase MD, Brunetti ND. Pleiotropic effects of statin in therapy in heart failure: a review. Curr Vasc Pharmacol. 2014;12(6):873-84.

28. Gastelurrutia P, Lupon J, de Antonio M, Urrutia A, Diez C, Coll R, et al. Statins in heart failure: the paradox between large randomized clinical trials and real life. Mayo Clinic proceedings. 2012;87(6):555-60.

29. Sola S, Mir MQ, Rajagopalan S, Helmy T, Tandon N, Khan BV. Statin therapy is associated with improved cardiovascular outcomes and levels of inflammatory markers in patients with heart failure. Journal of cardiac failure. 2005;11(8):607-12. 
30. Anker SD, Clark AL, Winkler R, Zugck C, Cicoira M, Ponikowski P, et al. Statin use and survival in patients with chronic heart failure--results from two observational studies with 5200 patients. International journal of cardiology. 2006;112(2):234-42.

31. Alehagen U, Benson L, Edner M, Dahlstrom U, Lund LH. Association between use of statins and outcomes in heart failure with reduced ejection fraction: prospective propensity score matched cohort study of 21864 patients in the Swedish Heart Failure Registry. Circulation Heart failure. 2015;8(2):252-60.

32. Mozaffarian D, Nye R, Levy WC. Statin therapy is associated with lower mortality among patients with severe heart failure. The American journal of cardiology. 2004;93(9):1124-9.

33. Dickinson MG, Ip JH, Olshansky B, Hellkamp AS, Anderson J, Poole JE, et al. Statin use was associated with reduced mortality in both ischemic and nonischemic cardiomyopathy and in patients with implantable defibrillators: mortality data and mechanistic insights from the Sudden Cardiac Death in Heart Failure Trial (SCD-HeFT). American heart journal. 2007;153(4):573-8.

34. Liu G, Zheng XX, Xu YL, Lu J, Hui RT, Huang XH. Effects of Lipophilic Statins for Heart Failure: A Meta-analysis of 13 Randomised Controlled Trials. Heart, lung \& circulation. 2014.

35. Preiss D, Campbell RT, Murray HM, Ford I, Packard CJ, Sattar N, et al. The effect of statin therapy on heart failure events: a collaborative meta-analysis of unpublished data from major randomized trials. European heart journal. 2015;36(24):1536-46.

36. Wang JQ, Wu GR, Wang Z, Dai XP, Li XR. Long-term clinical outcomes of statin use for chronic heart failure: a meta-analysis of 15 prospective studies. Heart, lung \& circulation. 2014;23(2):105-13.

37. Rauchhaus M, Coats AJ, Anker SD. The endotoxin-lipoprotein hypothesis. Lancet. 2000;356(9233):930-3.

38. Marz W, Siekmeier R, Muller HM, Wieland H, Gross W, Olbrich HG. Effects of lovastatin and pravastatin on the survival of hamsters with inherited cardiomyopathy. Journal of cardiovascular pharmacology and therapeutics. 2000;5(4):275-9.

39. Moosmann B, Behl C. Selenoprotein synthesis and side-effects of statins. Lancet. 2004;363(9412):892-4. 
40. Araujo JP, Frioes F, Azevedo A, Lourenco P, Rocha-Goncalves F, Ferreira A, et al. Cholesterol--a marker of nutritional status in mild to moderate heart failure. International journal of cardiology. 2008;129(1):65-8.

41. Sheng X, Wei L, Murphy MJ, MacDonald TM. Statins and total (not LDL) cholesterol concentration and outcome of myocardial infarction: results from a meta-analysis and an observational study. Eur J Clin Pharmacol. 2009;65(11):1071-80.

42. Murphy MJ, Wei L, Watson AD, MacDonald TM. 'Real-life' reduction in cholesterol with statins, 1993 to 2002. Br J Clin Pharmacol. 2008;65(4):587-92.

43. Bonsu KO, Reidpath DD, Kadirvelu A. Effects of Statin Treatment on Inflammation and Cardiac function in Heart Failure: An Adjusted Indirect Comparison Meta-analysis of Randomised Trials. Cardiovasc Ther. 2015.

44. Brunetti ND, Correale M, Totaro A, Ferraretti A, Monaco I, Passero T, et al. Lower cardiovascular mortality with atorvastatin and rosuvastatin vs simvastatin: Data from "moderateintensity" statin users in an observational registry on chronic heart failure (Daunia Heart Failure Registry). International journal of cardiology. 2015;194:23-7. 


\section{Figure legends}

\section{Figure 1}

Title: Kaplan-Meier curves for 10-year survival for chronic heart failure outpatients with respect to statin treatment

Legend: Small numbers indicate the numbers at risk at the respective follow-up.

\section{Figure 2:}

Title: Forrest plot for Cox regression analyses for all-cause mortality in the general cohort, in patients with statin treatment and in patients without statin treatment with respect to total serum cholesterol levels.

Legend: $\mathrm{TC}_{1}$, total serum cholesterol $\leq 3.6 \mathrm{mmol} / \mathrm{L} ; \mathrm{TC}_{2}$, total serum cholesterol $3.7-4.9 \mathrm{mmol} / \mathrm{L} ; \mathrm{TC}_{3}$, total serum cholesterol 5.0-6.2mmol/L; $\mathrm{TC}_{4}$, total serum cholesterol $>6.2 \mathrm{mmol} / \mathrm{L}$; HR, hazard ratio; CI, confidence interval.

\section{Figure 3 a) and b)}

Title: Kaplan-Meier curves for 10-year survival for chronic heart failure outpatients a) with statin treatment, and b) without statin treatment with respect to total serum cholesterol levels.

Legend: $\mathrm{TC}_{1}$, total serum cholesterol $\leq 3.6 \mathrm{mmol} / \mathrm{L} ; \mathrm{TC}_{2}$, total serum cholesterol $3.7-4.9 \mathrm{mmol} / \mathrm{L} ; \mathrm{TC}_{3}$, total serum cholesterol $5.0-6.2 \mathrm{mmol} / \mathrm{L} ; \mathrm{TC}_{4}$, total serum cholesterol $>6.2 \mathrm{mmol} / \mathrm{L}$.Small numbers indicate the numbers at risk at the respective follow-up. 


\section{Figure 4:}

Title: Cox regression analyses for all-cause mortality in the matched cohort, in matched patients with statin treatment and in matched patients without statin treatment with respect to total serum cholesterol levels.

Legend: $\mathrm{TC}_{1}$, total serum cholesterol $\leq 3.6 \mathrm{mmol} / \mathrm{L} ; \mathrm{TC}_{2}$, total serum cholesterol $3.7-4.9 \mathrm{mmol} / \mathrm{L} ; \mathrm{TC}_{3}$, total serum cholesterol 5.0-6.2 $\mathrm{mmol} / \mathrm{L} ; \mathrm{TC}_{4}$, total serum cholesterol $>6.2 \mathrm{mmol} / \mathrm{L} ; \mathrm{HR}$, hazard ratio; CI, confidence interval.

\section{Figure 5 a) and b)}

Title: Kaplan-Meier curves for 10-year survival for matched chronic heart failure outpatients a) with statin treatment and b) without statin treatment with respect to total serum cholesterol levels.

Legend: $\mathrm{TC}_{1}$, total serum cholesterol $\leq 3.6 \mathrm{mmol} / \mathrm{L} ; \mathrm{TC}_{2}$, total serum cholesterol $3.7-4.9 \mathrm{mmol} / \mathrm{L} ; \mathrm{TC}_{3}$, total serum cholesterol $5.0-6.2 \mathrm{mmol} / \mathrm{L} ; \mathrm{TC}_{4}$, total serum cholesterol $>6.2 \mathrm{mmol} / \mathrm{L}$. Small numbers indicate the numbers at risk at the respective follow-up.

\section{Figure 6}

Title: Probability of five-year death in matched chronic heart failure patients with respect to total serum cholesterol levels and statin treatment 
Table 1: Baseline characteristics for the complete cohort and separate with respect to statin treatment

\begin{tabular}{|c|c|c|c|c|}
\hline Characteristic & $\begin{array}{l}\text { All patients } \\
\qquad(\mathrm{n}=2,992)\end{array}$ & $\begin{array}{l}\text { No statin } \\
(\mathrm{n}=\mathbf{1 , 7 8 3})\end{array}$ & $\begin{array}{c}P \text { - } \\
\text { value }\end{array}$ & $\begin{array}{c}\text { Statin } \\
(\mathrm{n}=1,209)\end{array}$ \\
\hline Age, years & $63.8 \pm 15.0$ & $62.2 \pm 16.2$ & $<0.001$ & $65.5 \pm 12.9$ \\
\hline Male, $n(\%)$ & $2,025(67.7)$ & $1,176(66.0)$ & 0.014 & $849(70.2)$ \\
\hline Aetiology, $n(\%)$ & & & 0.25 & \\
\hline $\mathrm{DCM}$ & $1,569(52.4)$ & $953(53.4)$ & & $616(51.0)$ \\
\hline Hypertensive & $383(12.8)$ & $233(13.1)$ & & $150(12.4)$ \\
\hline Valvular & $260(8.7)$ & $156(8.7)$ & & $104(8.6)$ \\
\hline Other & $780(26.1)$ & $441(24.7)$ & & $339(28.0)$ \\
\hline NYHA, $n(\%)$ & & & 0.003 & \\
\hline I & $659(22.2)$ & 418 (23.7) & & $241(20.2)$ \\
\hline II & $1,505(50.8)$ & $914(51.8)$ & & $591(49.4)$ \\
\hline III & $777(26.2)$ & $422(23.9)$ & & 355 (29.7) \\
\hline IV & $21(0.7)$ & $12(0.7)$ & & $9(0.8)$ \\
\hline $\mathrm{SBP}, m m H g$ & $124 \pm 22$ & $124 \pm 23$ & 0.42 & $125 \pm 22$ \\
\hline $\mathrm{HR}, 1 / \min$ & $70 \pm 14$ & $71 \pm 15$ & 0.015 & $70 \pm 13$ \\
\hline BMI, $k g / m^{2}$ & $27.3 \pm 5.4$ & $27.1 \pm 5.5$ & 0.015 & $27.6 \pm 5.3$ \\
\hline LVEF, \% & $34 \pm 14$ & $35 \pm 14$ & 0.19 & $34 \pm 14$ \\
\hline \multicolumn{5}{|l|}{ Co-morbidity } \\
\hline $\mathrm{aHT}, n(\%)$ & $1,303(43.5)$ & $738(41.4)$ & 0.004 & $565(46.7)$ \\
\hline $\mathrm{COPD}, n(\%)$ & $489(16.3)$ & $285(16.0)$ & 0.52 & $204(16.9)$ \\
\hline Diabetes, $n(\%)$ & $449(15.0)$ & $215(12.1)$ & $<0.001$ & $234(19.4)$ \\
\hline Smoker, $n(\%)$ & $432(14.4)$ & $249(14.0)$ & 0.45 & $183(15.1)$ \\
\hline Country, $n(\%)$ & & & $<0.001$ & \\
\hline Norway & $1,616(54.0)$ & $878(49.2)$ & & $738(61.0)$ \\
\hline Germany & $893(29.8)$ & $562(31.5)$ & & $331(27.4)$ \\
\hline
\end{tabular}


UK

NTproBNP, pmol/L

Creatinine, $\mu \mathrm{mol} / \mathrm{L}$

eGFR, $\mathrm{mL} / \mathrm{min} / 1.73 \mathrm{~m}^{2}$

Sodium, mmol/L

Potassium, mmol/L

BUN, $\mu \mathrm{mol} / \mathrm{L}$

TC, $\mathrm{mmol} / \mathrm{L}$

TC, $n(\%)$

$\leq 3.6 \mathrm{mg} / \mathrm{dL}$

$3.7-4.9 \mathrm{mg} / \mathrm{dL}$

5.0-6.2 mg/dL

$>6.2 \mathrm{mg} / \mathrm{dL}$

Treatment

Aspirin, $n(\%)$

ACEI, $n(\%)$

ACEI dose equivalent,

$\%$

$\mathrm{ARB}, n(\%)$

ACEI and/or ARB, $n$

(\%)

ACEI/ARB dose

equivalent, \%

Beta blocker, $n(\%)$

Beta blocker dose

equivalent, $\%$
483 (16.1)

135

(31-555)

91

(77-113)

$70(53-88)$

$139 \pm 9$

$4.4 \pm 0.5$

405 (321-500)

$5.0 \pm 1.3$

436 (14.6)

$1,058(35.4)$

$1,029(34.4)$

469 (15.7)

744 (24.9)

2,111 (70.6)

$100(50-100)$

$600(20.1)$

$2,614(87.4)$

75 (50-100)

$2,429(81.2)$

$53(26-100)$
343 (19.2)

157

(31-724)

90

(75-113)

0.024

$71(54-90)$

$139 \pm 11$

0.013

$139 \pm 5$

$4.3 \pm 0.5$

0.004

$4.4 \pm 0.5$

$399(315-506)$

0.35

$411(327-494)$

$$
5.2 \pm 1.3
$$

$<0.001$

$4.8 \pm 1.3$

$<0.001$

$$
216(12.1)
$$

$220(18.2)$

$570(32.0)$

$488(40.4)$

682 (38.3)

347 (28.7)

315 (17.7)

$154(12.7)$

$359(20.1) \quad<0.001$

385 (31.8)

$1,238(69.4)$

0.10

$873(72.2)$

$100(50-100) \quad 0.07 \quad 100(50-100)$

$344(19.3)$

0.22

$256(21.2)$

$1,529(85.8) \quad 0.001$

$1,080(89.3)$

$62.5(50-100) \quad 0.06 \quad 75(50-100)$

$1,390(78.0) \quad<0.001 \quad 1,039(85.9)$

$53(26-100) \quad 0.054 \quad 53(26-100)$ 
Aldosterone antagonist,

$945(31.6)$

$558(31.3)$

0.22

$387(32.1)$

$n(\%)$

Loop diuretic, $n(\%)$

$2,100(70.2)$

$1,226(68.8)$

0.038

$874(72.3)$

Loop diuretic dose, $m g$

$40(40-80) \quad 40(40-80) \quad 0.51 \quad 40(40-80)$

furosemide

DCM, dilated cardiomyopathy; NYHA, New York Heart Association functional class; SBP, systolic blood pressure; HR, heart rate; BMI, body mass index; LVEF, left ventricular ejection fraction; aHT, arterial hypertension; COPD, chronic obstructive pulmonary disease; UK, United Kingdom; eGFR, estimated glomerular filtration rate using the Modification of Diet in Renal Disease equation; BUN, blood urea nitrogen; TC, total serum cholesterol; ACEI, angiotensin converting enzyme inhibitor; ARB, angiotensin receptor blocker. Italic nnumbers indicate significant $\mathrm{p}$-values $(\mathrm{p}<0.05)$. 
Table 2: Baseline characteristics for matched patients and separate with respect to statin treatment

\begin{tabular}{|c|c|c|c|c|}
\hline Characteristic & $\begin{array}{l}\text { Matched patients } \\
\qquad(\mathrm{n}=1,736)\end{array}$ & $\begin{array}{l}\text { No statin } \\
(\mathbf{n}=\mathbf{8 6 8})\end{array}$ & $P$-value & $\begin{array}{l}\text { Statin } \\
(n=868)\end{array}$ \\
\hline Age, years & $64.6 \pm 14.2$ & $63.8 \pm 15.2$ & 0.02 & $65.4 \pm 13.0$ \\
\hline Male, $n(\%)$ & $1,188(68.4)$ & $581(66.9)$ & 0.18 & 607 (69.9) \\
\hline Aetiology, $n(\%)$ & & & 0.89 & \\
\hline $\mathrm{DCM}$ & $906(52.2)$ & $449(51.7)$ & & 457 (52.6) \\
\hline Hypertensive & $210(12.1)$ & $104(12.0)$ & & $106(12.2)$ \\
\hline Valvular & $153(8.8)$ & $81(9.3)$ & & $72(8.3)$ \\
\hline Other & 467 (26.9) & $234(27.0)$ & & $233(26.8)$ \\
\hline NYHA, $n(\%)$ & & & 0.05 & \\
\hline I & $333(19.2)$ & $147(16.9)$ & & $186(21.4)$ \\
\hline II & $914(52.6)$ & $483(55.6)$ & & $431(49.7)$ \\
\hline III & $480(27.6)$ & $233(26.8)$ & & 247 (31.6) \\
\hline IV & $9(0.5)$ & $5(0.6)$ & & $4(0.5)$ \\
\hline SBP, $m m H g$ & $123 \pm 22$ & $123 \pm 22$ & 0.67 & $123 \pm 21$ \\
\hline $\mathrm{HR}, 1 / \min$ & $70 \pm 14$ & $70 \pm 14$ & 0.87 & $70 \pm 13$ \\
\hline BMI, $k g / m^{2}$ & $27.2 \pm 5.3$ & $27.2 \pm 5.5$ & 0.91 & $27.2 \pm 5.0$ \\
\hline LVEF, \% & $33 \pm 13$ & $34 \pm 13$ & 0.83 & $34 \pm 13$ \\
\hline \multicolumn{5}{|l|}{ Co-morbidity } \\
\hline $\mathrm{aHT}, n(\%)$ & $799(46.0)$ & $392(45.2)$ & 0.47 & 407 (46.9) \\
\hline COPD, $n(\%)$ & $277(16.0)$ & $139(16.0)$ & 0.95 & $138(16.0)$ \\
\hline Diabetes, $n(\%)$ & $273(15.7)$ & $134(15.4)$ & 0.74 & $139(16.0)$ \\
\hline Smoker, $n(\%)$ & $258(14.9)$ & $129(14.9)$ & 1.00 & $129(14.9)$ \\
\hline Country, $n(\%)$ & & & 0.03 & \\
\hline Norway & 1,061 (61.1) & $516(59.4)$ & & $545(62.8)$ \\
\hline Germany & $455(26.2)$ & $224(25.8)$ & & $231(26.6)$ \\
\hline
\end{tabular}


UK

NTproBNP, pmol/L

Creatinine, $\mu \mathrm{mol} / \mathrm{L}$

eGFR, $\mathrm{mL} / \mathrm{min} / 1.73 \mathrm{~m}^{2}$

Sodium, $\mathrm{mmol} / \mathrm{L}$

Potassium, $\mathrm{mmol} / \mathrm{L}$

BUN, $\mu \mathrm{mol} / \mathrm{L}$

$\mathrm{TC}, \mathrm{mmol} / \mathrm{L}$

TC, $n(\%)$

$\leq 3.6 \mathrm{mmol} / \mathrm{L}$

3.7-4.9 mmol/L

4.9-6.2 $\mathrm{mmol} / \mathrm{L}$

$>6.2 \mathrm{mmol} / \mathrm{L}$

Treatment

Aspirin, $n(\%)$

ACEI, $n(\%)$

ACEI dose equivalent,

$\%$

$\mathrm{ARB}, n(\%)$

ACEI and/or ARB, $n$

(\%)

ACEI/ARB dose

equivalent, $\%$

Beta blocker, $n(\%)$

Beta blocker dose

equivalent, $\%$
$483(12.7)$

128

$(35-475)$

$91(78-114)$

$69(52-86)$

$139 \pm 3$

$4.4 \pm 0.5$

406

(327-500)

$4.9 \pm 1.2$

$274(15.8)$

$684(39.4)$

549 (31.6)

$229(13.2)$

$502(28.9)$

$1,315(75.7)$

$100(50-100)$

$351(20.2)$

$1,602(92.3)$

809 (93.2)

0.31

$798(91.9)$

$139(16.0)$

$339(39.1)$

$276(31.8)$

$114(13.1)$

0.97

0.99

135 (15.6)

345 (39.7)

$273(31.5)$

$115(13.2)$

$246(28.3) \quad 0.60$

$256(29.5)$

$661(76.2)$

0.70

$654(75.3)$

$100(50-100)$

0.84

$100(50-100)$

173 (19.9)

0.77

$178(20.5)$

$100(50-100)$

$100(50-100)$

0.74

$100(50-100)$

$1,524(87.8)$

767 (88.4)

0.46

757 (87.2)

$53(26-100)$

$53(26-100)$

0.28

$53(38-100)$ 
Aldosterone antagonist, $n(\%)$

$586(33.8)$

$297(34.2)$

0.66

$289(33.3)$

Loop diuretic, $n(\%)$

$1,265(72.9)$

$635(73.2)$

0.79

$630(72.6)$

Loop diuretic dose, $m g$

furosemide

$40(40-80)$

0.68

$40(40-80)$

DCM, dilated cardiomyopathy; NYHA, New York Heart Association functional class; SBP, systolic blood pressure; HR, heart rate; BMI, body mass index; LVEF, left ventricular ejection fraction; aHT, arterial hypertension; COPD, chronic obstructive pulmonary disease; UK, United Kingdom; eGFR, estimated glomerular filtration rate using the Modification of Diet in Renal Disease equation; BUN, blood urea nitrogen; TC, total serum cholesterol; ACEI, angiotensin converting enzyme inhibitor; ARB, angiotensin receptor blocker. Italic numbers indicate significant $\mathrm{p}$-values $(\mathrm{p}<0.05)$ 